\title{
The "landscape" of Pati-Salam heterotic superstring vacua
}

\author{
J. Rizos ${ }^{1, *}$ \\ ${ }^{1}$ Theory Division, Physics Department, University of Ioannina, 45110 Ioannina, Greece
}

Key words string phenomenology, Pati-Salam model, exotics, fractionally charged states.

The main aspects of a recently developed method for classification of heterotic superstring vacua in the Free Fermionic Formulation are presented. Using these techniques we classify a big number of approximately $10^{15}$ heterotic string vacua with Pati-Salam, $S U(4) \times S U(2)_{L} \times S U(2)_{R}$, gauge symmetry with respect to their main phenomenological features as the number of families, Pati-Salam breaking Higgs, Standard Model Higgs doublets, additional triplets and exotic charge states. We identify an interesting subclass of these vacua, approximately one to one million, whose massless spectrum is completely free of fractionally charge states.

Copyright line will be provided by the publisher

\section{Introduction}

String theory provides a framework for unification of gauge interactions with gravity. There exist only five string theories in 10 dimensions, the $E_{8} \times E_{8}$ and $S O(32)$ heterotic, the type I, type IIA and type IIB strings. However, compactification leads to a plethora of string vacua in four dimensions. Recently, a lot of effort has been devoted in studying the properties of these vacua, also referred as "string landscape" [1], including intersecting D-brane orientifold models [2] and heterotic models [3, 6].

In references [5, 6] a new approach has been developed that allows to study a big class of heterotic vacua, with $S O(10)$ embedding, in the context of Free Fermionic Formulation [4]. A model in this class is defined by a set of 12 basis vectors

$$
\begin{aligned}
v_{1}=1 & =\left\{\psi^{\mu}, \chi^{1, \ldots, 6}, y^{1, \ldots, 6}, \omega^{1, \ldots, 6} \mid \bar{y}^{1, \ldots, 6}, \bar{\omega}^{1, \ldots, 6}, \bar{\eta}^{1,2,3}, \bar{\psi}^{1, \ldots, 5}, \bar{\phi}^{1, \ldots, 8}\right\} \\
v_{2}=S & =\left\{\psi^{\mu}, \chi^{1, \ldots, 6}\right\} \\
v_{2+i} & =e_{i}=\left\{y^{i}, \omega^{i} \mid \bar{y}^{i}, \bar{\omega}^{i}\right\}, i=1, \ldots, 6 \\
v_{9} & =b_{1}=\left\{\chi^{34}, \chi^{56}, y^{34}, y^{56} \mid \bar{y}^{34}, \bar{y}^{56}, \bar{\eta}^{1}, \bar{\psi}^{1, \ldots, 5}\right\} \\
v_{10}=b_{2} & =\left\{\chi^{12}, \chi^{56}, y^{12}, y^{56} \mid \bar{y}^{12}, \bar{y}^{56}, \bar{\eta}^{2}, \bar{\psi}^{1, \ldots, 5}\right\} \\
v_{11}=z_{1} & =\left\{\bar{\phi}^{1, \ldots, 4}\right\} \\
v_{12}=z_{2} & =\left\{\bar{\phi}^{5, \ldots, 8}\right\}
\end{aligned}
$$

and a set of 66 phases $c\left[v_{i}, v_{j}\right]= \pm 1, j<i=1, \ldots, 12$, that correspond to generalized GSO projections (GGSO). The basis vectors $b_{1}, b_{2}$ correspond to $Z_{2} \times Z_{2}$ orbifold twists, while $e_{i}, i=1, \ldots, 6$ correspond to shifts. Keeping the basis vectors fixed and varying the GGSO phases we can generate $2^{12(12-1) / 2}=2^{66}$ models with $S O(10) \times U(1)^{3} \times S O(8)^{2}$ gauge symmetry. Following [6] we can derive expressions for the main phenomenological aspects of the model in terms of the GGSO phases. The number of twisted

* Presented at School and Workshops on Cosmology and Strings: Theory-Cosmology-Phenomenology (CORFU2009), September 6-13, Corfu , Greece. 
spinorial representations, per orbifold plane $I=1,2,3$, is given by

$$
\#\left(S^{(I)}\right)=\left\{\begin{array}{ll}
2^{4-\operatorname{rank}\left(\Delta^{(I)}\right)} & \operatorname{rank}\left(\Delta^{(I)}\right)=\operatorname{rank}\left[\Delta^{(I)}, Y_{16}^{(I)}\right. \\
0 & \operatorname{rank}\left(\Delta^{(I)}\right)<\operatorname{rank}\left[\Delta^{(I)}, Y_{16}^{(I)}\right.
\end{array}\right]
$$

while the number of vectorial representations is given by

$$
\#\left(V^{(I)}\right)=\left\{\begin{array}{ll}
2^{4-\operatorname{rank}\left(\Delta^{(I)}\right)} & \operatorname{rank}\left(\Delta^{(I)}\right)=\operatorname{rank}\left[\Delta^{(I)}, Y_{10}^{(I)}\right. \\
0 & \operatorname{rank}\left(\Delta^{(I)}\right)<\operatorname{rank}\left[\Delta^{(I)}, Y_{10}^{(I)}\right.
\end{array}\right]
$$

where $\Delta^{(I)}$ are $4 \times 4$ and $Y^{(I)}$ are $4 \times 1$ GGSO coefficient matrices and $\left[\Delta^{(I)}, Y_{10}^{(I)}\right],\left[\Delta^{(I)}, Y_{16}^{(I)}\right]$ represent the enchased $4 \times 5$ matrices. Similar expressions can be derived for the chirality of spinorial representations. Using these results a full classification of $S O(10)$ vacua, numbering approximately $10^{20}$ models, has been presented in [6], revealing some interesting properties of the spectra as the spinor-vector duality.

\section{The Pati-Salam superstring "landscape"}

In this work we report some recent progress in extending the analysis of [6] by adding an extra basis vector to (1) that breaks the $S O(10)$ symmetry. The simplest choice is the vector

$$
v_{13}=\alpha=\left\{\bar{\psi}^{45}, \bar{\phi}^{1,2}\right\}
$$

that introduces 12 new GSO projection phases $c\left[\alpha, v_{j}\right], j=1, \ldots, 12$. The full gauge group (away from special points) is broken down to $S U(4) \times S U(2)_{L} \times S U(2)_{R} \times U(1)^{3} \times S U(2)^{4} \times S O(8)$ which includes the Pati-Salam as the symmetry of the "observable" sector [8].

A heterotic string realization of a supersymmetric version of the Pati-Salam model has been studied in [9]. The SM particles are accommodated in PS multiplets

$$
\begin{aligned}
& S U(4) \times S U(2)_{L} \times S U(2)_{R} \supset S U(3) \times S U(2) \times U(1) \\
& F_{L}(\mathbf{4}, \mathbf{2}, \mathbf{1}) \rightarrow Q\left(\mathbf{3}, \mathbf{2},-\frac{1}{6}\right)+\ell\left(\mathbf{1}, \mathbf{2}, \frac{1}{2}\right) \\
& \bar{F}_{R}(\overline{\mathbf{4}}, \mathbf{1}, \mathbf{2}) \rightarrow u^{c}\left(\overline{\mathbf{3}}, \mathbf{1}, \frac{2}{3}\right)+d^{c}\left(\overline{\mathbf{3}}, \mathbf{1},-\frac{1}{3}\right)+e^{c}(\mathbf{1}, \mathbf{1},-1)+\nu^{c}(\mathbf{1}, \mathbf{1}, 0) \\
& D(\mathbf{6}, \mathbf{1}, 1) \rightarrow D_{3}\left(\mathbf{3}, \mathbf{1}, \frac{1}{3}\right)+\bar{D}_{3}\left(\overline{\mathbf{3}}, \mathbf{1},-\frac{1}{3}\right) \\
& h(\mathbf{1}, \mathbf{2}, \mathbf{2}) \rightarrow h^{d}\left(\mathbf{1}, \mathbf{2}, \frac{1}{2}\right)+h^{u}\left(\mathbf{1}, \mathbf{2},-\frac{1}{2}\right)
\end{aligned}
$$

where $D_{3}, \bar{D}_{3}$ are additional quark triplets. Using the standard hypercharge embedding, that guarantees $\sin ^{2} \theta_{w}=\frac{3}{8}$ at the string scale, $Y=\frac{1}{\sqrt{16}} T_{15}+\frac{1}{2} I_{3 L}+\frac{1}{2} I_{3 R}$, states transforming as $(\mathbf{1}, \mathbf{1}, \mathbf{2}),(\mathbf{1}, \mathbf{2}, \mathbf{1})$, $(4,1,1)$ accommodate fractional charge exotics. The appearance of these states is a generic feature of $k=1$ string compactifications as shown in [10].

The PS symmetry can be broken to the Standard Model by vevs to the neutral components $\left(\left\langle\nu_{H}^{c}\right\rangle,\left\langle\nu_{H}\right\rangle\right)$ of a pair of Higg multiplets $(H, \bar{H})$

$$
\begin{aligned}
& \bar{H}(\overline{\mathbf{4}}, \mathbf{1}, \mathbf{2}) \rightarrow u_{H}^{c}\left(\overline{\mathbf{3}}, \mathbf{1}, \frac{2}{3}\right)+d_{H}^{c}\left(\overline{\mathbf{3}}, \mathbf{1},-\frac{1}{3}\right)+\nu_{H}^{c}(\mathbf{1}, \mathbf{1}, 0)+e_{H}^{c}(\mathbf{1}, \mathbf{1},-1) \\
& H(\mathbf{4}, \mathbf{1}, \mathbf{2}) \rightarrow u_{H}\left(\mathbf{3}, \mathbf{1},-\frac{2}{3}\right)+d_{H}\left(\mathbf{3}, \mathbf{1}, \frac{1}{3}\right)+\nu_{H}(\mathbf{1}, \mathbf{1}, 0)+e_{H}(\mathbf{1}, \mathbf{1}, 1)
\end{aligned}
$$


The triplet remnants of the Higgs mechanism get heavy masses combined with the triplets in $D(\mathbf{6}, \mathbf{1}, 1)$ through the superpotential terms

$$
H^{2} D+\bar{H}^{2} D \rightarrow d_{H} \bar{D}_{3}\left\langle\nu_{H}\right\rangle+d_{H}^{c} D_{3}\left\langle\nu_{H}^{c}\right\rangle
$$

Fermion masses are generated by the superpotential term

$$
F_{L}(\mathbf{4}, \mathbf{2}, \mathbf{1}) \bar{F}_{R}(\overline{4}, \mathbf{1}, 2) h(\mathbf{1}, \mathbf{2}, 2)
$$

however neutrinos stay naturally light as they mix with additional heavy singlets [9].

Following the method developed in [6], analytic formulae are derived for the main features of a PS model massless spectrum in terms of GGSO coefficients. These include the number of $F_{L}, \bar{F}_{L}, F_{R}, D, \bar{D}, H$, $\bar{H}, h$ as well as the numbers of exotic multiplets. After fixing some coefficients to guarantee $N=1$ supersymmetry, and removing some redundancy, we are left with 51 independent phases giving rise to a class of $2^{51} \sim 10^{15}$ vacua. Using a computer program and random sampling over $5 \times 10^{9}$ models we obtain a statistical profile of this class of models. In Figure 1 we plot the number of PS models versus the number of fermion generations. We remark that 3 -generation vacua correspond approximately to $0.3 \%$ of the sample. Furthermore, in Figure 2, we plot the number of models versus the number of exotic multiplets in the spectrum. We observe that an important number of models in the sample, approximately one PS model per half milion, is free of massless exotics. This phenomenologically interesting result is not in contradiction with [10], since exotics are still present in the massive heterotic string spectrum. A detail analysis of the "landscape" of PS heterotic vacua is presented in [7]. An outline of the main results is shown in Table 11. where we examine a series of phenomenological constraints and calculate the statistics of the models satisfying them. Every line in the table corresponds to an additional constraint. We remark that the number of vacua satisfying all requirements, is approximately one to one million. This reveals a rich subclass of phenomenologically interesting vacua that deserve further study.

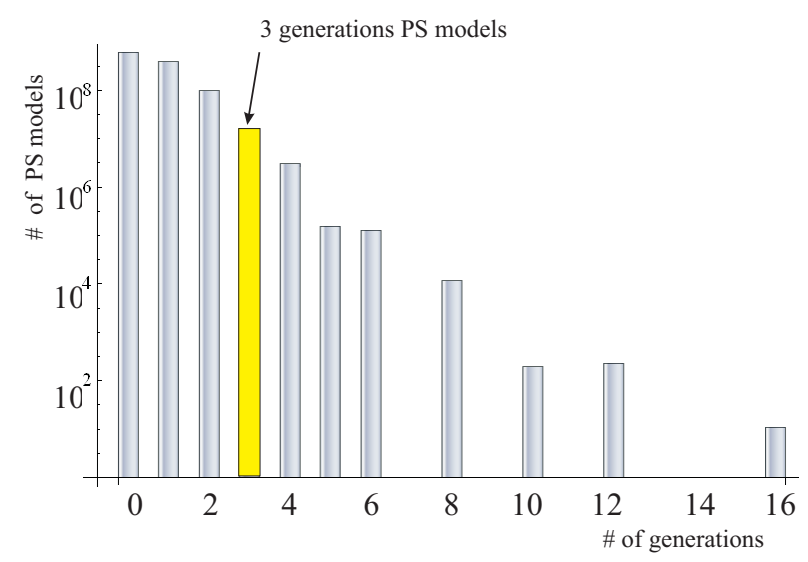

Fig. 1 Number PS models versus number of generations in a random sample of $5 \times 10^{9}$ models.

Acknowledgements This work is supported in part by the EU under contract MRTNCT20060358631. The author would like to thank University of Liverpool and CERN Theory Division for the hospitality.

\section{References}

[1] see e.g:

M.R. Douglas, JHEP 0305(2003)046;

F. Denef and M.R. Douglas, JHEP 0405(2004)072;

M.R. Douglas and W. Taylor, JHEP 0701(2007)031. 


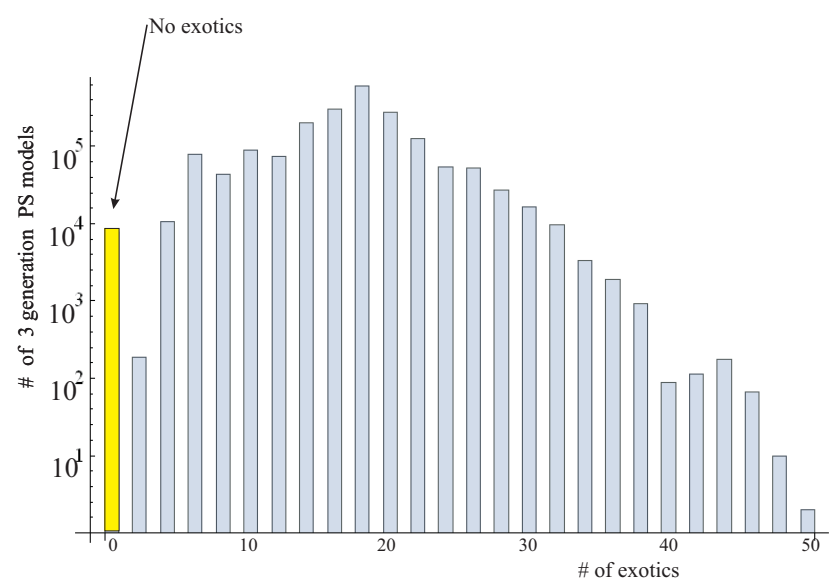

Fig. 2 Number 3 generation PS models versus number number of exotic state multiplets in a random sample of $5 \times 10^{9}$ models.

\begin{tabular}{llcc}
\hline \hline & Constraint & probability & estimated \# of models \\
\hline \hline & No gauge group enhancements & $2 \times 10^{-1}$ & $2 \times 10^{14}$ \\
\hline+ & 3 generation models & $3 \times 10^{-3}$ & $7 \times 10^{12}$ \\
\hline+ & PS breaking Higgs & $4 \times 10^{-4}$ & $9 \times 10^{11}$ \\
\hline+ & SM breaking Higgs doublets & $3 \times 10^{-4}$ & $7 \times 10^{11}$ \\
\hline+ & No exotics & $1 \times 10^{-6}$ & $2 \times 10^{9}$ \\
\hline
\end{tabular}

Table 1 PS models statistics with respect to phenomenological constraints imposed on massless spectrum.

[2] see eg:

T.P.T. Dijkstra, L. Huiszoon and A.N. Schellekens, Nucl. Phys. B 710(2005) 3;

B.S. Acharya, F. Denef and R. Valadro, JHEP 0506(2005)056;

P. Anastasopoulos, T. P. T. Dijkstra, E. Kiritsis and A. N. Schellekens, Nucl. Phys. B 759 (2006) 83;

R. Blumenhagen, F. Gmeiner, G. Honecker, D. Lust and T. Weigand, Nucl. Phys. B 713 (2005) 83;

F. Gmeiner, R. Blumenhagen, G. Honecker, D. Lust and T. Weigand, JHEP 0601 (2006) 004;

E. Kiritsis, M. Lennek and B. Schellekens, Nucl. Phys. B 829 (2010) 298.

[3] D. Senechal, Phys. Rev. D 39 (1989) 3717;

K. R. Dienes, Phys. Rev. D 73 (2006) 106010;

O. Lebedev, H. P. Nilles, S. Ramos-Sanchez, M. Ratz and P. K. S. Vaudrevange, Phys. Lett. B 668 (2008) 331;

F. Gmeiner, R. Blumenhagen, G. Honecker, D. Lust and T. Weigand, JHEP 0601 (2006) 004.

[4] I. Antoniadis, C. Bachas, and C. Kounnas, Nucl. Phys. B289 (1987) 87;

H. Kawai, D.C. Lewellen, and S.H.-H. Tye, Nucl. Phys. B288 (1987) 1;

I. Antoniadis and C. Bachas, Nucl. Phys. B298 (1988) 586.

[5] A. Gregori, C. Kounnas and J. Rizos, Nucl. Phys. B549 (1999) 16.

[6] A. E. Faraggi, C. Kounnas, S. E. M. Nooij and J. Rizos, proceedings of 2nd International Conference on String Phenomenology 2003, Durham, England, 29 Jul - 4 Aug 2003, [arXiv:hep-th/0311058];

A. E. Faraggi, C. Kounnas, S. E. M. Nooij and J. Rizos, Nucl. Phys. B 695 (2004) 41;

A. E. Faraggi, C. Kounnas and J. Rizos, Phys. Lett. B 648 (2007) 84; Nucl. Phys. B 774 (2007) 208; Nucl. Phys. B 799 (2008) 19;

T. Catelin-Jullien, A. E. Faraggi, C. Kounnas and J. Rizos, Nucl. Phys. B 812 (2009) 103.

[7] B. Assel, K. Christodoulides, A. E. Faraggi, C. Kounnas and J. Rizos, Phys. Lett. B 683 (2010) 306

[8] J. C. Pati and A. Salam, Phys. Rev. D 10 (1974) 275 [Erratum-ibid. D 11 (1975) 703].

[9] I. Antoniadis and G. K. Leontaris, Phys. Lett. B 216 (1989) 333;

I. Antoniadis, G. K. Leontaris and J. Rizos, Phys. Lett. B 245 (1990) 161;

G. K. Leontaris and J. Rizos, Nucl. Phys. B 554 (1999) 3.

[10] A. N. Schellekens, Phys. Lett. B 237 (1990) 363. 